NORTH

AMERICAN

PRIMARY CARE From the North American

GROUP

Primary Care Research Group

Ann Fam Med 2010;8:471-472. doi:10.1370/afm.1182.

\section{Involving Community Residency Programs in Research: The Residency Research Network of Texas}

For research to be a core value, it cannot remain the province of a small cadre of our members. ${ }^{1}$

For years, family medicine leaders have been urging members to increase research productivity and promote a strong research culture. Still, research inhabits a tiny corner of the family medicine world, concentrated in academic centers. Most residents (84\%) graduate from community residency programs, and most of these lack the resources to promote research among faculty and residents. How can we expect our graduates to appreciate, support, and participate in research when the great majority has little exposure?

In the middle 1990s, these concerns led us to examine our own back yard. While our university faculty were research-friendly, our department had done little to spread these values beyond our walls. To correct this situation, we applied to the US Health Resources and Services Administration (HRSA) and received funding in 1997, 2000, 2003, and 2009 to support the Residency Research Network of Texas (RRNeT). We proposed a two-fold mission: to examine health issues relevant to family medicine patients in Texas; and to increase community program faculty and residents' involvement in research.

\section{Infrastructure}

RRNeT held its first meeting in March 1998 with 5 residency programs; membership now includes 10 programs. Together, our programs employ about 100 family physician faculty and train 290 residents per year. Our clinicians see approximately 300,000 outpatient visits per year, including 60\% Latino, 15\% AfricanAmerican, and 22\% white patients. RRNeT membership requires the endorsement and commitment of the program director, who designates 1 or more faculty to represent the program on the RRNeT Steering Committee. RRNeT communicates via list-serve frequently, by conference call every 2 months, and face-to-face during 2 meetings per year.

Funding is modest. HRSA provides salary support to the Director and Coordinator at the University of Texas Health Science Center at San Antonio
(UTHSCSA), and funds travel to RRNeT meetings and national conferences for dissemination. The Texas Academy of Family Medicine, the Office of the Medical Dean at UTHSCSA, and the Area Health Education Center (AHEC) provide support for student research assistants. Currently, RRNeT has a pilot grant from the UTHSCSA Center for Translational Science Award to support a study about chronic low back pain.

\section{Process}

RRNeT conducts about 1 project per year, on average. The turnaround is quick, about 1 year from idea generation to data analysis. Participation is informal, collaborative, and non-mandatory, but programs rarely opt out. Our network prefers research questions that come from community program clinicians, not from university investigators. The Steering Committee meets to brainstorm and prioritize research ideas, then develops a skeleton research protocol through large group discussion. A "content expert" is identified and recruited onto the planning team (if not already a member); then 1 or 2 individuals draft the protocol which provides the format for IRB applications and grant proposals. Our 10 programs have 10 IRBs, so we develop model protocols and consent forms; Steering Committee members are responsible for adapting these documents for their own institution.

RRNeT Steering Committee members oversee data collection at their sites. We have used varying methods, including patient and physician surveys, "pocket cards," chart reviews, and qualitative long interviews. Data are entered into a central database, cleaned, and translated into SPSS (Statistical Package for the Social Sciences). Data files are "owned" by the network and shared with Steering Committee members on request. Data analysis is usually (but not always) conducted by the network Director at UTHSCSA, with assistance from departmental statisticians. Dissemination has 4 steps: findings first go to the Steering Committee; then to the practices; to national conferences; and finally as published journal articles.

\section{Outcomes}

Over 12 years, RRNeT has examined several topics: complementary and alternative medicine use ; quality of diabetes care; brief interventions for medication adherence and for safe firearm storage; preventive care for adolescents; health behaviors; management of nonmalignant chronic pain; chronic low back pain; and stories of efficiencies in the health care system. We have published 6 journal articles and 7 abstracts; presented 32 presentations to national conferences; and won 8 research awards, 3 to medical students and 5 to community residency faculty. 


\section{Conclusions}

RRNeT provides a centralized university-based infrastructure, a link to research resources, and an engine to move projects forward. This allows busy clinical teachers to participate actively in research while maintaining their curricular and patient care responsibilities The benefits to community programs include: a peer group of researchers; links to career investigators and statisticians; hands-on research experience; opportunities to present and publish; national exposure for the training programs; and documentation for involvement in research for accreditation purposes. The advantage to university faculty is a large and diverse patient population with a thoughtful, collaborative team of clinical teachers. Now, THIS is science!

Sandra K. Burge, PbD, Professor, Department of Family \& Community Medicine Director,

Residency Research Network of Texas, University of Texas Health Science Center at San Antonio

\section{References}

1. Rosenthal MP, Dickinson PW, Bogdewic SP, Sherwood RA. The state of family medicine research, or, stop, hey, what's that sound? Everybody look what's going down! Fam Med. 2002;34(1):48-50.

\section{SAFP}

Ann Fam Med 2010;8:472. doi:10.1370/afm.1178.

\section{Final Definition of 'Meaningful Use' of EHRs Modified Based on AAFP Comments}

The AAFP's response to the federal government's December 30, 2009 release of electronic health record (EHR) regulations that define the term "meaningful use" has resulted in significant modifications to 2 final rules that define and support the meaningful use of EHRs.

In a February 26, 2010 letter to CMS Acting Administrator Charlene Frizzera, AAFP Board Chair Ted Epperly, MD, of Boise, Idaho, detailed how the agency could make the regulations more helpfuland more palatable-to family physicians. Epperly noted, "We believe that certain aspects in the details of these regulations are unworkable, excessive, or redundant and will actually impede the very goals of the legislation."

One rule, which was issued by the Office of the National Coordinator for Health Information Technology, identifies the standards and certification criteria for the certification of EHR technology so that hospi- tals and physicians will know that the EHRs in which they invest can perform the required functions.

A companion rule, issued by CMS, defines the minimum EHR meaningful use objectives that physicians and other professionals must meet to qualify for bonus programs enacted under the American Recovery and Reinvestment Act of 2009.

According to a July 13, 2010 HHS news release, CMS estimates it may pay out $\$ 27$ million in incentive payments during the next 10 years. Physicians and other health care professionals can choose to participate in the Medicare bonus program-potentially earning as much as $\$ 44,000$ in additional income-or they can choose to earn Medicaid incentives of as much as $\$ 63,750$.

When the 2 final rules were announced at the July 13, 2010 press conference hosted by Health and Human Services Secretary Kathleen Sebelius, the AAFP was gratified to learn that some of the Academy's biggest concerns had been addressed. According to Steven Waldren, MD, director of the AAFP's Center for Health IT, "CMS has addressed the Academy's biggest concerns, and many of the changes they have made will benefit family physicians."

For example, in its February 26, 2010 comment letter to CMS, the AAFP suggested that CMS abandon its "all-or-nothing" approach to achieving meaningful use of EHRs and, instead, increase physician participation by offering partial incentives. Waldren pointed out that the final rule grants physicians greater flexibility in meeting and reporting certain objectives for demonstrating meaningful use. The final regulation states that physicians initially must meet 15 core measures but then can choose from a subset of additional objectives to work on in 2011-2012.

"CMS also significantly reduced the threshold for some of the measures that the AAFP deemed particularly onerous for physicians," said Waldren. For example, the threshold for an electronic prescribing measure that originally called for $75 \%$ of all permissible prescriptions to be transmitted electronically has been reduced to $40 \%$.

Another priority issue for the Academy was CMS' measure on computerized provider order entry that called for transmitting at least $80 \%$ of all orders electronically. That percentage now applies to medication orders only, and the threshold for electronic transmission of other types of orders has been reduced, said Waldren.

CMS received more than 2,000 comments on the proposed meaningful use rule. Waldren noted that staff members in the Center for Health IT staff will carefully analyze the meaningful use rule and the certification rule to make sure that they understand what members have to do to qualify. 